\title{
Implementation of an EMR System for a Comprehensive Dental Service within a Large Regional Hospital Network: Challenges and Opportunities Presented by the Introduction of new Technology
}

\section{Stephen Swanik*}

Division of Health Policy and Administration, Public Health Informatics Program, School of Public Health, University of Illinois at Chicago and Departments of Medical Education and Dentistry at Advocate Illinois Masonic Medical Center, Chicago, IL.

\begin{abstract}
Objectives: The development of new information technology has significant effects on the health care system, and its implementation and the associated change management process can bring some positive changes and gains in understanding, but there are challenges with making the transition. These benefits and challenges are explored in the context of a hospital based dental department. Additionally, the concept of the integration of oral health to overall systemic health is explored in context with an Electronic Medical Records system implementation, and the American Dental Association's recent recognition of dental anesthesiology as a clinical subspecialty.

Method: Qualitative survey of attending dental faculty members of the department, who represent a broad range of dental specialties and experience in private practice, hospital based practice, teaching, and public health practice.

Results: The faculty survey yielded some consistent themes, ranging from enhanced information to make better diagnoses, to challenges in transitioning to EMR, as well as concerns about data security and too much time and effort in front of a computer screen.

Discussion: A brief summary of the history of the stand-alone development of dentistry is given, which contributed to the separate development of dental EMRs from hospital EMRs. The various modalities of clinical care provided by the Department of Dentistry at Advocate Illinois Masonic Medical Center, Chicago, IL are presented to give a scope of the areas of need a successful EMR solution must meet in a hospital based dental setting. Public health aspects are included in the discussion.

Conclusion: Macro level health data sets (ie NHANES, state level datasets) have the potential to be expanded to include more thorough data, combining medical health data and oral health data in the same datasets.

*Correspondence: sswani3@uic.edu, steve.swanik@advocatehealth.com, sswanik15@yahoo.com
\end{abstract}


DOI: 10.5210/ojphi.v11i2.10131

Copyright (C2019 the author(s)

This is an Open Access article. Authors own copyright of their articles appearing in the Online Journal of Public Health Informatics. Readers may copy articles without permission of the copyright owner(s), as long as the author and OJPHI are acknowledged in the copy and the copy is used for educational, not-for-profit purposes.

\section{Introduction}

This writing will take a look at the considerations that go along with implementation of an electronic dental record system in a hospital based environment, with a discussion on the implications for public health that can come from a higher quality data yield that can result from a model consistent with integration of oral health and overall medical/systemic health. The department featured is the Department of Dentistry at Advocate Illinois Masonic Medical Center in Chicago, IL, USA, a teaching hospital in the non-profit Advocate-Aurora health system. This is a large regional hospital network consisting of 27 hospitals and 500 sites of care across the states of Illinois and Wisconsin. The dental department at Illinois Masonic is the only full clinical dental department in this entire health system, and it is anticipating a transition to the Epic Wisdom dental EMR platform in 2020. The department currently uses paper charts for clinical records and an electronic system for scheduling and billing.

\section{Department background and brief history: teaching/education and public health components}

The dental department was originally started in 1970 as a means to provide dental care for special needs children, benefiting from a close relationship with Masonic charities through its affiliation with Illinois Masonic hospital. It developed a postgraduate General Practice Residency dental training program (GPR), and a dental practice. Presently, the dental department is on the same standing in the hospital as the other clinical departments. It provides emergency on call services for the hospital, provides dental consults to inpatients, and sees patients with other medical comorbidities that make it medically risky for them to receive dental care in stand-alone dental practices. In 2000, it launched a mobile dental van program, providing dental care to a diverse set of sites including Chicago Public Schools, Thresholds Psychiatric Rehabilitation Centers, and others. In 2016, the department aligned with the neighboring Howard Brown Health Center, an FQHC with a focus on providing healthcare to the LGBT community, to provide routine and complex dental care for its patient population. The department is launching a residency training program in dental anesthesiology, the newest recognized dental specialty [1] in July, 2019. When Advocate Health Care and Aurora Care merged in 2018, system leadership decided to implement Epic throughout the entire system. As of this writing, the dental department is slated to convert from paper based records to Epic Wisdom in June 2020. 
The department has had significant historical challenges with respect to its conversion to EMR, yet it enjoys very broad success with its teaching programs and its public health programs. The Department houses a longstanding postgraduate dental General Practice Residency (GPR) training program and will be launching the training program in dental anesthesiology in July 2019. At any given time, there are approximately 35 attending members of the department, most of whom have part time involvement ranging from activity such as teaching the residents a half a day a month, or as clinicians who perform dental cases in the hospital's operating room. The GPR houses 9 residents and is a one year program in length. The dental anesthesiology program will house two residents per year and is a 3 year long training program. The structure of the department, considering its public health practice, its relationship with its hospital, and its upcoming conversion to an EMR system lends to it being a good example with respect to strategically planning for public health utility of an EMR system. It can additionally serve as a good example of dynamic change management in process, with positive takeaways and errors to learn from.

The department has programs to provide care to different population modalities. It has a longstanding Special Patient Dental Care program, providing dental care for special needs patients, often times under IV Sedation provided by a dental anesthesiologist and the dental residents. It has a mobile dental van which serves a variety of sites, including schools, assisted living facilities, psychiatric centers, and others on a monthly rotating basis. It also has a partnership with Howard Brown Health Center, a local FQHC which provides care primarily to Chicago's large LGBT community, to provide oral health care to its patient population. The launch of the dental anesthesiology program will additionally provide an avenue to increase departmental capacity to provide care for special needs patients.

\subsection{Major Population Groups Served/sociodemographic analysis}

The department serves a diverse patient population, and has many programs in place to provide services to a diverse variety of underserved patients. The major population groups that are served by the department, in addition to a cohort of general PPO patients seeking routine dental care and hospital employees are as follows:

The Special Patient Dental Care program

--This program, as its name implies, provides dental care to special needs patients.

These conditions include cerebral palsy, autism spectrum disorder, and others. Many of these patients are treated under IV Sedation guided by a dental anesthesiologist, Dr. Ken Kromash. Additionally, this is a significant component of training for the dental residents. With the upcoming addition of a dental anesthesiology training program, the department is anticipating increasing its capacity to provide care to special needs patients, particularly special needs children.

HBHC and LGBT population

--In 2016, Howard Brown partnered with the dental department to provide dental care for its patients. Some of these patients are HIV +, some are transgender, 
and many of them are LGBT. Other literature exists [2,3] on the considerations of providing culturally competent care for the LGBT community, and this partnership aims to meet those ends. The HBHC partnership provides approximately $25 \%$ of the patient population of the clinic.

Medically compromised patients

--The department, being hospital based, assumes oral health responsibility for the hospital inpatients. It sees medically compromised patients who require dental care that may be unsafe to provide in a private practice dental setting due to the medical condition of the patient. An example of this modality would be an inpatient requiring a complicated anesthetic intubation who receives a preoperative dental clearance or dental treatment from the dental department before the other medical treatment.

Mobile van populations

--The department's mobile van populations include a variety of patients from a variety of sites. Some of the partnerships exist merely to meet a socioeconomic need, but some sites, such as psychiatric and geriatric sites, may present a coexisting medical need as well.

Each of these populations, for one reason or another, presents a potential need for clinical care that is a union of both medical care and dental care.

\subsection{History and Current Status of EMR Conversion}

There have been two previous unsuccessful attempts to convert the department to EMR in the last ten years. The most recent was an attempt at a conversion to Eaglesoft, a major dental EMR program in the market. The vendor and hospital technical teams were in the process of resolving security compatibility of a dental software program that was not developed with a primary focus of being implemented in a large hospital system, and the business contracts were in the process of being developed, signed, and executed. This project was aborted when Advocate Health Care merged with Aurora Health Care, and the decision was made to convert all Advocate EMR systems to Epic Systems, Inc. - meaning of course that the dental program became contractually obliged to utilize Epic Wisdom, the dental module for the Epic software platform. This transition is now scheduled to occur in 2020. The challenges associated with security capability can be traced at least somewhat back to the concept of the historical division of oral health from overall systemic health. As dental EMR software systems were initially designed, the concept of implementing them into hospital-based environments was not the first priority, and conversely when hospital EMRs were developed, the dental/oral health components were not the first priority.

A previous attempt in around 2014 - 2015 also failed, but this failure was specifically at the departmental level, as clinicians and administrative management were unable to successfully collaborate and agree on an EMR system. What was particularly telling about this is that the postgraduate residents in the educational training program, though highly wanting to utilize EMR, strongly objected to the chosen product, in part because "It is apparent that this system was chosen based on convenience and the long-term relationship built with a current vendor. We feel that even 
though the institution wants to respect a long-term relationship with the vendor, the software offered does not satisfy the needs of the organization."

\section{Intersection between EMR system and physician}

There already exists significant literature on the topic of the relationship between the physician, the patient and the EMR system. Challenges that still exist include the fact that many physicians feel burdened by certain aspects of the EMR system, as examples they spend much more time performing administrative tasks, and many of them feel that during patient encounters they are spending too much time looking at a computer screen and not interacting with patients. Schulte and Fry recently published a summary [4] of this conversation in Medscape. Some of these challenges were reported in a survey conducted of the department teaching faculty.

\section{Brief Summary of the History of the Divide Between Oral Health and Systemic Health in the US}

Another factor to look at is the relationship between oral health and systemic health in the U.S. health system. This has been a longstanding "fait accompli" in the US. However there are current initiatives to move towards a more full integration of oral health and systemic health, as biologically these two systems are fully intact and interact with each other - a dental abscess leading to an infection serving as a routine example.

Mertz wrote a summary [5] in 2016 of how we got to where we are today in Health Affairs. Some of the highlights of this include the historical routes of "barber-surgeons," "distinct from physicians, nurses, and pharmacists." As such, dental schools were independently established after the development of medical schools. Dental insurance was not originally packaged with health or medical insurance and was developed as a separate product line from medical insurance. The clinical delivery system was designed separately and distinctly from medical systems; dental practice and hygiene teams have their own separate training apparatuses. And though the Affordable Care Act took a stab towards pediatric dental coverage, it did not significantly address dental coverage for adults. Mertz also touches a key point for this writing, that "electronic dental records are rarely interoperable with medical records, limiting oral and systemic health research, and dentistry lacks a fully deployed set of diagnostic codes, stymieing comparative effectiveness research."

Simon advocates in the AMA Journal of Ethics [6] for integration and increased collaboration as a way to promote health equity, mentioning areas in which physician training can incorporate areas of oral health education, and vice versa, but does not cover the role of an EMR.

The American Dental Association additionally has invested some resources exploring the integration of oral health with overall systemic health and has produced literature on this topic. Its Chief Economist, Dr. Marko Vujicic, wrote an editorial [7] in the Journal of the American Dental Association about the topic. Some key topics he addressed include defining and systematically measuring oral health, and reforming the care delivery model. 
An area of high significance with respect to both oral and medical care is the presence and slated expansion of dental anesthesiology in the department. In 2016, Giovannitti et al wrote a detailed summary of the specialty [8] and its history. This article covers a brief history of the discovery of anesthesia, and the clinical relationship between dentistry and anesthesia. It also gives a history of the development of dental anesthesiology training programs, and a detailed and thorough explanation of the educational standards. It then goes into a discussion of need, growth, and challenges of the discipline. As the topic of this article was about the specialty of dental anesthesia, and highly focused on clinical training standards, it did not address EMR systems. An analogy can be made however between the clinical integration of oral and medical health brought about by dental anesthesiology and the digital integration of digital oral health and digital medical health brought about by an EMR system encapsulating both.

A key takeaway from this writing is that an integrated EMR can be used as a tool to drive the integration, and that this integrated knowledge is beneficial for patient care and ultimately public health.

\section{Survey of dental faculty}

There are approximately 35 faculty members on the medical staff at AIMMC, and they were all asked to participate in a voluntary survey about their experiences and thoughts with EMR. The survey focused on the public health aspects of an EMR and the clinicians' thoughts about data interoperability. The goal was to obtain a minimum of 10 quality responses, and this was achieved. The faculty provided many high quality comments, and these responses converged over some major themes and reinforced the necessity to further address those themes - particularly themes such as user friendliness, data security.

The survey questionnaire was developed with the help of Drew Gripentrog, DMD, who is an alum of the GPR program and a current attending faculty member. There were 12 responses, and these responses yielded some consistent themes.

These were the questions asked in the questionnaire:

1. What in your opinion are the most important features/characteristics of an EMR system itself?

2. Do you currently use an EMR in private practice? Is it cloud based?

3. Have you ever been involved in the history of an implementation/conversion in either a private practice or a hospital/school-based environment? What are some key takeaways from that experience?

4. What do you find to be the most important oral health considerations that EMR can help to identify or address?

5. Do you see any key public health insights that can be derived from EMR?

6. Do you notice any trends among population groups with respect to any specific oral health concerns - whether those population groups are age, income, ethnicity, geographic locations, or others? How does EMR facilitate identifying trends? 
7. What characteristics do you most hope to see in the future dental EMRs?

8. How significant do you find data transmission between sites or other colleagues to be with respect to care? Things such as patient referrals, transfer of x-rays from one sight to another sight? What type of information transfer do you find the most important to be for effective care?

9. If you could integrate your private practice's EMR and its respective data with any hospital systems you are affiliated with, would you? What do you see as potential strengths of this? What concerns would you have?

10. Has the opioid crisis affected your clinical practice and if so how?

Here are some selected responses with some comments:

"I converted my private pediatric dental practice in the Chicago suburbs from a paper based charting system and computerized billing (on an antiquated dental software program) with digital radiography to a completely paperless integrated system (EMR). It took 6 months, I had to purchase a new \$10,000 server, and lots of extra manpower to scan all that paper and convert the accounts from one computer system to another. Choose the new software system wisely (do your research for ease of use and conversion support), be sure your IT people are aware of your storage needs first, and be sure your office staff have proper training, motivation, and take ownership of the project. Set goals and achieve them."

This is a response to question 6, aligning with the concept of utilization of EMR to pull reports for public health purposes.

"Yes - perio in the hispanic population. A lot of enamel hypoplasia in our geographic location. Yes - much easier to pull reports. This is also good for financials and seeing public health trends"

This comment was in response to question 7, regarding future potentials of EMRs. An interesting catch with respect to the medical history and how further development can still come for more accurate diagnostic information in medical histories.

"Compatibility with their physicians would be amazing so that the medical history wouldn't be self reported - instead we could see what is really going on and what medications they are taking, how well they are controlled, etc."

Another survey:

"Identification of oral habits can be correlated to race and culture, caries risk assessment and anticipatory guidance can be quite related to social economical status." 
"It will be very beneficial if any oral health provider could somehow communicate within the EMR system regardless of the hospital network they belong to."

This comment ties into the large historical conversation of IT development and interoperability as it relates to the proprietary features of computer systems: historically Apple products and Microsoft pcs and products couldn't talk to one another - and this change has started to become more commonplace in large part due to consumer demand and expectation. This perhaps may be area where health care can learn from history in other segments. If the system develops so that EMRs establish a de factor oligopoly, then the question of proprietary rights and effective transmission of data across competitors will need to be addressed.

Another survey:

"Yes. Data collection. Being able to monitor disease incidence, prevalence, and treatment outcomes. Being able to determine quality measures for value. Being able to look at trends over time. Being able to share information with non-medical folks who can help with things like nutritional counseling, smoking cessation programs, etc."

"I hope to see a way to integrate with medical EMR systems. I hope to be able to refer directly to other colleagues like behavioral therapists, occupational therapists, physical therapists, nutritionists, drug and smoking cessation counselors, pharmacists. I'd want those colleagues to see what dental treatments we were providing and why. I'd want my dental treatment to need a diagnostic code so other providers can know why I'm performing certain treatments. I'd want my dental diagnostic code to have to link to a medical code when necessary. For example, if I've diagnosed periodontal disease, I'd want a medical diagnostic code that connects when relevant. For example, if a patient has periodontal disease and diabetes, those therapies should link together somehow in the EMR so that we can track the outcomes of treatment."

This comment is a very broad comment, touching on many important aspects, with an emphasis on coordination of care.

This respondent also comments on data transferability, from the perspective of providing complete care to patients: "It's necessary but not a modern system. You'd think that as easy as it is to take a photo on my phone and share it with the world automatically, I should be able to transfer data seamlessly without having to email attachments, etc. If I'm treating a dental patient, everything 
that I do, any images, etc, should automatically be in the patient's medical record and accessible. The physician should get a notification when patients are visiting the dentist and vice versa."

Another response re-emphasized the importance of transferability of data:

"The most important feature of any EMR system is the ease of portability of the records. I think that someday in the future, patients will be the owners and maintainers of their own health records and they can take them with them wherever they go. It is theoretically easier for doctors/hospitals to transfer records digitally if the information is available. If patients are the keepers of their own health records, and it is stored digitally on a chip, then in an emergency, the records can be available even if the patient is not able to communicate. That can save lives."

"I think it will be easier to identify population health statistics from EMR.”

"Among my patient population, across all the categories you cited, people want to save their teeth and have them looking good. Nobody likes flossing! EMR can facilitate identifying the trends because all the parameters are readily available within a software program."

The theme of the difficulty of making the EMR systems compatible with physician workflow was also mentioned:

"I want them more user friendly so I don't have to click as many boxes or open as many windows to make an entry."

"It is easier and often faster to get patient records. That can impact patient care, especially when you have the patient in the office and you need a record from another office immediately. The most important information I have found to be useful for patient care has been images, either radiographic or otherwise." - conversation about which information is most important for clinicians

"I don't know if I would integrate my EMR data with a hospital system. There would be security concerns. Also, I think the only time it would be truly useful is when there is a crossover between medicine and dentistry, such as cancer of the head and neck. Potential strengths are better care of the patient."

"The biggest surprise that I have from EMR is that I thought it would make my life easier and I would be spending less time on records. It is completely the opposite. I am spending more time on them and it 
is making the practice of health care much less enjoyable. And now I have to worry about data security a lot more. There a many more ways PHI can be obtained with an EMR than with a paper record."

There were some key responses regarding conversion to EMR.

"Yes, was involved in transitioning from paper charts to EMR in dental school. The experience was pretty bumpy- some faculty even chose to retire over it instead of learning the new system. They tried to implement "superusers" who were trained ahead of time, but there were either not enough of these, or these users didn't really know how to work the software either. I think a soft release would've helped, but we all went live on the day they picked and no one really did their homework of knowing how to work it. A lot of money was lost with not walking out the correct procedures or knowing how to bill. It was a big hassle."

"In my private practice, I converted from paper charts to EMR. The key takeaways for me were: 1) You just have to make the change. No looking back--just walk to the edge and jump! 2) It will be stressful, but there are advantages. 3) It won't make your life that much easier. 4) I am spending more time on records now than I was before. 5) It costs a lot 6) There are more threats to security with EMR than with paper."

"If EMR can help to shorten appointment lengths, or make sure to remind patients of appointments and keep them on a recall schedule, then that is very useful. It also makes charting and keeping an accurate history of visits and services much easier and clearer." This comment addressed the theme of the utility of EMRs and digital systems for simplifying and reducing error in basic routines.

"EMR over time should be able to pick up trends in services provided or reasons for visits. Good EMR should be able to run reports looking at almost any variable, be is by service, or zip code, or utilization, etc. The possibilities are endless, and this is something you just couldn't do with paper charting."

This respondent was aware of a common theme in information technology circles, "Garbage in, Garbage out," or "GIGO."

"People tend to be wanting implants more, but this also varies greatly with SES. People tend to be wanting full mouth makeovers i.e. big veneer cases less (due to economic conditions/uncertainty) 
Again, a good EMR should be able to run almost any report over a defined period of time with respect to procedure codes and zip code. Unfortunately you don't usually have income data, but you do have age, race, ethnicity, zip code, etc. However, EMR reports are only as good as the data and accuracy of data put in."

"I would love to be able to transfer a prescription electronically to any pharmacy. I would love to be able to send an xray or CBCT scan electronically and securely through EMR. Getting records from another office is usually a sadly difficult thing to do. I would love to be able to click and send over a digital referral to a specialist and know they got it." This is an important comment, particularly as the clinical discipline of dentistry is heavily dependent on the general dentist's ability to develop a specialty referral network, and to efficiently communicate patient need between specialists.

"This is very difficult usually. Either the patient forgets, the e-mail gets lost, the other office forgets, sometimes the requests are denied, etc. It's almost always easier to just take your own new xrays. Even if you get previous xrays, sometimes the quality is so poor due to the format, they are essentially useless."

Question 9, the question about integrating a private practice's dental records with a hospital system, yielded some interesting responses as well.

"I don't think integrating my private dental practice with a hospital system in particular would be that useful, but maybe if there was a generic third party portal to transfer data on an as needed basis that could be useful."

"I understand that EPIC, the hospital's computer system, has a dental component but no one uses it. Typically I use EPIC to write Operative reports for patients treated under general anesthesia in the OR or emergency patients in the ER. If we need to transfer information to a physician's office, we send it via encrypted email. If we need to access something from EPIC, the practice owner has remote access in his office. I don't think MDs would understand our charting systems and dental procedures (in our dental software or in the dental portion of EPIC) enough for it to be beneficial without our interpretation."

"Yes! Absolutely. The only concerns would be that we would end up seeing too much - but really everything is applicable since we take care of the whole person." 


\section{Public Health Considerations}

There already are existing sources for oral health data. Examples of these include the Dental Quality Alliance [9] (DQA), affiliated with the ADA, and the Center for Disease Control's National Health and Nutrition Examination Survey (NHANES). The CDC additionally has a website [10] set up to explore oral health data by location, https://www.cdc.gov/oralhealthdata/. The state of Illinois Department of Public Health has oral health resources as well [11]. However, most of these are stand-alone data sets focusing essentially exclusively on oral health, and as such they do not completely capture the corresponding medical status of the populations in the same way as a fully-integrated medical record consisting of both medical data and oral health data could.

The opportunity for the future is the upcoming potential to capture and use the population level data and metadata generated from hospital based dental EMR systems and other to be developed systems to elucidate further understanding. Oral health information can now be directly linked to and keyed with corresponding medical data, allowing for more opportunities clinical research, and to inform policy makers, advocates, and other stakeholders with strategies to improve overall health. Examples such as the link between periodontitis and cardiovascular health can now be much further explored, as can demographic, and even geographic based data be generated.

\section{Concluding Remarks}

Dental EMR Conversions have happened before, and literature [12] already exists about the challenges associated with this. Now, we take the next steps by looking at the integration of a dental EMR in a hospital based environment. This distinction opens up the discussion to other important areas beyond just the technical specifics of an EMR system. These areas include the applications and broader utility that can come from the use of the implemented system as a tool to provide insight into the systemic integration of oral health into total health. An integrated EMR is posited as a tool to better increase population health. As hospital based dental practices are equipped to provide oral health to a variety of dentally underserved populations the subsequent aggregated metadata resulting from this treatment can in the future be used to broaden the public health discourse.

Many of the survey comments show that faculty are already inherently aware of many of the strengths and current challenges with EMR. Key concepts repeated multiple times include the benefit of facilitated aggregation and utility of population level data, and the subsequent generation of metadata enabled by deployment of EMR, yet the challenges of security, user friendliness, and the GIGO moniker are mentioned as well.

A key related takeaway is both the need and the potential for the development of a standardized dataset that contains both oral and medical aspects, as the currently existing oral health datasets are severely lacking commensurate medical data.

Dental anesthesia can serve as a clinical example of oral and systemic health integration - the utilization of anesthesia specifically for the dental context is a clinical example of this integration. 
With the recent specialty status recognition of dental anesthesia by the American Dental Association, this specialty is in a position to grow, and this development will also contribute to the conversation. This will be another area in which EMR systems can play a role.

That the dental department at Illinois Masonic is still using paper charts in its dental clinic is a clear indication of the importance of successful change management strategies, and the influence of multiple factors and needs affecting multiple stakeholders. From a certain perspective, it can be deemed problematic that a clinical department significantly involved with medical care does not have an EMR system in 2019. Nevertheless, this example shows the often surprising challenges that come with the development and implementation of new technology.

\section{Limitations}

The limitations in this writing stem from the fact that it explores only one department in one hospital system. Additionally, that department has yet to fully launch some key features -- the dental anesthesiology training program has a begin date of July 1, 2019, and the full EMR conversion is scheduled for June, 2020. There is an anticipated ramp up process in the future, however as of right now there has yet to be a critical mass of numbers developed for extended datasets discussed.

Secondly, the investigation for this was very focused on human experiences and interactions. Though valuable, they should be framed and taken into context as such. Individual experiences and perspectives are not empirically tested; they are only recorded and reported. Another important consideration is that only dentists were surveyed for this project, so the perspectives of computer engineers, software developers, and so forth are not covered. A next step could be to obtain insight from those personnel.

\section{Suggested Readings}

Schleyer, T., Song, M., Gilbert, G. H., Rindal, D. B., Fellows, J. L., Gordan, V. V., \& Funkhouser, E. (2013). Electronic dental record use and clinical information management patterns among practitioner-investigators in The Dental PracticeBased Research Network. The Journal of the American Dental Association, 144(1), 49-58.

Liu, K., Acharya, A., Alai, S., \& Schleyer, T. K. (2013). Using electronic dental record data for research: a data-mapping study. Journal of dental research, 92(7_suppl), S90-S96.

Acharya, A., Shimpi, N., Mahnke, A., Mathias, R., \& Ye, Z. (2017). Medical care providers' perspectives on dental information needs in electronic health records. The Journal of the American Dental Association, 148(5), 328-337.

Rindal, D. B., Kottke, T. E., Rush, W. A., Asche, S. E., \& Enstad, C. J. (2015, December). Implementation of an electronic dental record tool to increase referrals to a tobacco counseling quit line. In Implementation Science (Vol. 10, No. 1, p. A6). BioMed Central. 
Brent, B. K. (2018). A Survey of the Implementation and Usage of Electronic Dental Records and Digital Radiographs in Private Dental Practices in Mississippi.

Tokede, O., Ramoni, R. B., Patton, M., Da Silva, J. D., \& Kalenderian, E. (2016). Clinical documentation of dental care in an era of electronic health record use. Journal of Evidence Based Dental Practice, 16(3), 154-160.

Sheikhtaheri, A., Zarei, A., \& Ahmadi, M. (2018). A comparative study on the features of specialized electronic dental record systems. Journal of Health Administration (JHA), 20(70).

Sidek, Y. H., \& Martins, J. T. (2017). Perceived critical success factors of electronic health record system implementation in a dental clinic context: An organisational management perspective. International journal of medical informatics, 107, 88100.

Mertz, E. A. (2016). The dental-medical divide. Health Affairs, 35(12), 2168-2175.

Murphy, D. R., Meyer, A. N., Russo, E., Sittig, D. F., Wei, L., \& Singh, H. (2016). The burden of inbox notifications in commercial electronic health records. JAMA internal medicine, 176(4), 559-560.

Goh, W. P., Tao, X., Zhang, J., \& Yong, J. (2016). Decision support systems for adoption in dental clinics: a survey. Knowledge-Based Systems, 104, 195-206.

Yi, Y., Lee, J., Yi, H., Asher, S., Feldman, L., Rivas-Morello, C., ... \& Ross, E. (2015). Variables affecting general anesthesia time for pediatric dental cases. Pediatric dentistry, 37(7), 508-512.

Mandel, J. C., Kreda, D. A., Mandl, K. D., Kohane, I. S., \& Ramoni, R. B. (2016). SMART on FHIR: a standards-based, interoperable apps platform for electronic health records. Journal of the American Medical Informatics Association, 23(5), 899-908.

Goldstein, B. A., Navar, A. M., \& Pencina, M. J. (2016). Risk prediction with electronic health records: the importance of model validation and clinical context. JAMA cardiology, 1(9), 976-977.

Yüksel, B., Küpçü, A., \& Özkasap, Ö. (2017). Research issues for privacy and security of electronic health services. Future Generation Computer Systems, 68, 1-13.

Colaianni, C. A., Levesque, P. A., \& Lindsay, R. W. (2017). Integrating data collection into office work flow and electronic health records for clinical outcomes research. JAMA facial plastic surgery, 19(6), 528-532.

Kharrazi, H., Chi, W., Chang, H. Y., Richards, T. M., Gallagher, J. M., Knudson, S. M., \& Weiner, J. P. (2017). Comparing population-based risk-stratification model performance using demographic, diagnosis and medication data extracted from outpatient electronic health records versus administrative claims. Medical care, 55(8), 789-796.

Ritwik, P., Massey, C., \& Hagan, J. (2015). Epidemiology and outcomes of dental trauma cases from an urban pediatric emergency department. Dental traumatology, 31(2), 97-102. https://www.ncbi.nlm.nih.gov/pmc/articles/PMC1430363/

Macek, M. D., Manski, R. J., Vargas, C. M., \& Moeller, J. F. (2002). Comparing oral health care utilization estimates in the United States across three nationally 
representative surveys. Health services research, 37(2), 499-521. doi:10.1111/1475-6773.034

\section{References}

1. Solana K. (2019, March 12). ADA News: Anesthesiology recognized as a dental specialty. Retrieved April 20, 2019, from https://www.ada.org/en/publications/ada-news/2019archive/march/anesthesiology-recognized-as-a-dental-specialty

2. McEwing EM. 2017. Delivering Culturally Competent Care to the Lesbian, Gay, Bisexual, and Transgender (LGBT). Population.

3. Felsenstein DR. 2018. Enhancing Lesbian, Gay, Bisexual, and Transgender Cultural Competence in a Midwestern Primary Care Clinic Setting. J Nurses Prof Dev. 34(3), 142-50. doi:10.1097/NND.0000000000000450. $\underline{\text { PubMed }}$

4. Death by 1000 Clicks: Where EHRs Went Wrong. (2019, March 19). Medscape. Retrieved from

https://www.medscape.com/viewarticle/910608?nlid=128907_5204\&src=wnl_dne_190320_ mscpedit\&uac=145970PN\&impID=1912878\&faf $=1$

5. Mertz EA. 2016. The dental-medical divide. Health Aff. 35(12), 2168-75. PubMed https://doi.org/10.1377/hlthaff.2016.0886

6. Simon L. 2016. Overcoming historical separation between oral and general health care: interprofessional collaboration for promoting health equity. AMA J Ethics. 18(9), 941-49. PubMed https://doi.org/10.1001/journalofethics.2016.18.9.pfor1-1609

7. Vujicic M. 2018. Our dental care system is stuck: And here is what to do about it. J Am Dent Assoc. 149(3), 167-69. PubMed https://doi.org/10.1016/j.adaj.2018.01.006

8. Giovannitti JA, Montandon RJ, Herlich A. 2016. The Development of Dental Anesthesiology As a Discipline and Its Role As a Model of Interdisciplinary Collaboration. J Dent Educ. 80(8), 938-47. PubMed

9. (n.d.). Retrieved from https://www.ada.org/en/science-research/dental-quality-alliance/aboutdqa

10. Oral Health Data. (2018, July 27). Retrieved from https://www.cdc.gov/oralhealthdata/

11. Oral Health. (n.d.). Retrieved from http://www.dph.illinois.gov/topics-services/preventionwellness/oral-health 
12. Sidek YH, Martins JT. 2017. Perceived critical success factors of electronic health record system implementation in a dental clinic context: An organisational management perspective. Int J Med Inform. 107, 88-100. PubMed https://doi.org/10.1016/j.ijmedinf.2017.08.007 